\title{
Association of increased monetary cost of dietary intake, diet quality and weight management in Spanish adults
}

\author{
Helmut Schröder ${ }^{1,2 *}$, Luis Serra-Majem ${ }^{3,4}$, Isaac Subirana ${ }^{2,5}$, Maria Izquierdo-Pulido ${ }^{4,6}$, Montserrat Fitó ${ }^{1,4}$ \\ and Roberto Elosua ${ }^{5}$ \\ ${ }^{1}$ Cardiovascular Risk and Nutrition Research Group (CARIN), Hospital del Mar Medical Research Institute (IMIM), \\ O8003 Barcelona, Spain \\ ${ }^{2}$ CIBER Epidemiology and Public Health (CIBERESP), Instituto de Salud Carlos III, 28029 Madrid, Spain \\ ${ }^{3}$ Research Institute of Biomedical and Health Sciences, University of Las Palmas de Gran Canarias, 35016 Las Palmas, Spain \\ ${ }^{4}$ CIBER Physiopathology of Obesity and Nutrition (CIBEROBN), Instituto de Salud Carlos III, 28029 Madrid, Spain \\ ${ }^{5}$ Cardiovascular Epidemiology and Genetics Research Group (EGEC), Hospital del Mar Medical Research Institute (IMIM), \\ 08003 Barcelona, Spain \\ ${ }^{6}$ Department of Food Science and Nutrition, University of Barcelona, O8028 Barcelona, Spain
}

(Submitted 6 July 2015 - Final revision received 23 October 2015 - Accepted 16 November 2015 - First published online 13 January 2016)

\section{Abstract}

Higher monetary diet cost is associated with healthier food choices and better weight management. How changes in diet cost affect changes in diet quality and weight remains unknown. The aim of this study was to assess the impact of changes in individual monetary diet cost on changes in diet quality, measured by the modified Mediterranean diet score recommendations (MDS-rec) and by energy density (ED), as well as changes in weight and BMI. We conducted a prospective, population-based study of 2181 male and female Spaniards aged between 25 and 74 years, who were followed up to the 2009-2010 academic year. We measured weight and height and recorded dietary data using a validated FFQ. Average food cost was calculated from official Spanish government data. We fitted multivariate linear and logistic regression models. The average daily diet cost increased from 3.68 (SD 0.0.89)€/8.36 MJ to 4.97(SD 1.16)€/8.36 MJ during the study period. This increase was significantly associated with improvement in diet quality ( $\Delta$ ED and $\Delta$ MDS-rec; $P<0.0001)$. Each $1 €$ increase in monetary diet cost per $8.36 \mathrm{MJ}$ was associated with a decrease of $0.3 \mathrm{~kg}$ in body weight $(P=0.02)$ and $0.1 \mathrm{~kg} / \mathrm{m}^{2}$ in BMI $(P=0.04)$. These associations were attenuated after adjusting for changes in diet quality indicators. An improvement in diet quality and better weight management were both associated with an increase in diet cost; this could be considered in food policy decisions.

Key words: Diet cost: Diet quality: Weight gain: Prospective studies

A healthy diet is paramount for physical and mental health ${ }^{(1,2)}$, and improving population diets was declared a priority area of action at the United Nations High Level Meeting on Prevention and Control of Non-Communicable Diseases ${ }^{(3)}$. Diet quality depends on personal food choices, which are driven by food prices as well as by culture, taste and convenience ${ }^{(4)}$. Epidemiological evidence indicates that better diet quality is associated with higher diet costs ${ }^{(5)}$. Furthermore, higher price indices for fruits and vegetables were linked to higher BMI in children aged 2-9 years ${ }^{(6)}$.

From 2000 to 2010, diet costs increased disproportionately in European countries, with the greatest increases in South European countries such as Spain (31.2\%) compared with $17.2 \%$ in Germany or $20.6 \%$ in Sweden ${ }^{(7)}$. During that same decade, food prices rose more sharply in Spain for healthy food choices compared with less healthy foods ${ }^{(8)}$. The cost of foods low in energy density and rich in nutrients, such as fruits, increased by $51.0 \%$, whereas pastries or confectionery products, high in energy density but low in nutrient density, increased by 10.1 and $23.1 \%$, respectively. High-density energy consumption has been related to low nutrient adequacy ${ }^{(9,10)}$, weight gain $^{(11)}$ and risk of obesity ${ }^{(12)}$.

It is unknown how increases in individual diet cost, driven by rising food prices, affects consumers' food choices and, consequently, overall diet quality. Therefore, the aim of the present study was to analyse the prospective association between changes in individual diet cost and changes in diet quality in a representative Spanish population. In addition, we determined the impact of changes in diet cost on body weight.

Abbreviations: MDS-rec, modified Mediterranean diet score-recommended intake; rEI, reported energy intake.

* Corresponding author: Dr H. Schröder, fax +34933160 796, email hschroeder@imim.es 


\section{Methods \\ Participants}

Data were obtained from a population-based survey conducted in Girona (Spain) in 2000 and 2009. The baseline survey examined a randomly selected, population-based sample of 3058 men and women aged 25-74 years (participation rate: $71.0 \%$ ). Of the 3058 participants in the baseline survey in 2000, 2715 non-institutionalised participants who still resided in the catchment area in 2009 were invited to participate in the followup study (online Supplementary Fig. S1), and 2181 of these individuals attended the re-examination in 2009-2010. This represents a $19 \cdot 7 \%$ loss to follow-up after 10 years, resulting in an acceptable follow-up rate of $80 \cdot 3 \%$. Finally, $3 \cdot 2 \%$ ( $n$ 69) of the participants had missing dietary data at baseline or at follow-up and were excluded from the analysis. The final sample size included 2112 participants with complete follow-up data. Participants were duly informed and signed their consent to participate in the study. The project was approved by the local Ethics Committee (CEIC-PSMAR, Barcelona, Spain).

\section{Dietary assessment}

Food consumption was determined using a validated FFQ, administered by a trained interviewer at baseline and at follow-up ${ }^{(13,14)}$. In a 166-item food list including alcoholic and non-alcoholic beverages, participants indicated their usual consumption and chose from ten frequency categories ranging from never or less than once per month to six or more times per $\mathrm{d}$.

\section{Monetary diet cost}

Food prices were obtained from the food price database of the Spanish Ministry of Economy and Competitiveness ${ }^{(8)}$. The average prices for many food items (not including commercial fast foods) are updated every month in this database. For this study, we calculated food prices for 2000 and 2010, based on the average cumulated prices reported for each of those 2 years. Prices were not available for the following foods (2\%): paella, cannelloni and pizza. Prices for fast food items were obtained by a search of corporate websites. Individuals' daily diet cost and the monetary diet cost per $8.36 \mathrm{MJ}$ of energy intake/d (hereinafter, energy-adjusted diet cost) were calculated.

\section{Measurement of diet quality}

Diet quality was determined by adherence to the Mediterranean diet and by measuring the energy density of the daily diet. We chose these two indices of diet quality from among the numerous available indicators because of their good construct validity and established association with health outcomes ${ }^{(9-12,15-17)}$.

Modified Mediterranean diet score recommendations. Assessing adherence to the Mediterranean diet by a score based on population-based food consumption distribution is, by definition, specific to a particular population, making it difficult to compare results between studies. To overcome the limitation regarding comparability of results, we calculated the modified Mediterranean diet score recommendations (MDS-rec) as previously described $^{(18)}$. In brief, consumption that meets recommended intakes for Spanish adults of cereals, fruits, vegetables, legumes, fish, olive oil, nuts and dairy products is coded as 3, consumption at least weekly as 2 and less than weekly as 1 for legumes, fish and nuts; for the other groups (cereals, fruits, vegetables, olive oil, dairy products), consumption at least daily was coded as 2 and less than daily as 1 . For meat (including red meat, poultry and sausages) and dairy products, the score was partially inverted, with consumption more than weekly coded as 1, weekly as 2 and meeting the recommended consumption as 3. Moderate red wine consumption (up to $20 \mathrm{~g} / \mathrm{d}$ ) was coded as 3, and more or less than this daily portion was coded as 1 .

Energy density. After considering the different methods of calculating energy density ${ }^{(18)}$, we decided to present data on the basis of a dietary density calculation that includes only food items. Foods and beverages have different effects on satiety and energy intake, which in turn affects the association between energy density and body weight ${ }^{(19,20)}$. Therefore, total energy density of the diet was calculated by dividing total energy intake from food consumed each day by the total weight of the reported food intake.

\section{Anthropometrics}

Measurements were performed by a team of trained nurses and interviewers who used the same standard methods in both surveys. A precision scale of easy calibration was used for weight measurement with participants in underwear. Body weight was rounded up to the nearest $200 \mathrm{~g}$ and height was measured to the nearest $0.5 \mathrm{~cm}$. BMI was calculated by (weight $(\mathrm{kg}) /$ height squared $\left(\mathrm{m}^{2}\right)$ ). Body weight and BMI changes were defined as the difference between the weight and BMI recorded in 2010 and at baseline in 2000, respectively.

\section{Energy misreporting}

Individuals with implausible reported energy intake (rEI) were identified by the revised Goldberg method, as described previously $^{(21)}$. BMR was estimated using the Mifflin equation ${ }^{(22)}$. The rEI:BMR ratio was calculated. The plausibility of rEI was estimated by comparing the rEI:BMR ratio with physical activity levels (PAL). The cut-off values to identify plausible rEI were taken as the confidence limits of agreement between $\mathrm{rEI}$ :BMR and PAL, and were based on the CV of participants' energy intake, the accuracy of the BMR measurements and the total variation in PAL, as proposed by Black $^{(23)}$.

\section{Other variables}

The validated Minnesota Leisure-Time Physical Activity (LTPA) questionnaire $^{(24,25)}$ was administered by a trained interviewer. Smoking habits and demographic and socio-economic variables were obtained from structured standardised questionnaires administered by trained personnel. Participants were 
dichotomously categorised as non-smokers (never smokers and ex-smokers with more than 1 year of smoking cessation) and current smokers (including ex-smokers with less than 1 year of smoking cessation). Maximum education level attained was elicited and dichotomously recorded for analysis as primary school $v$. secondary school or university.

\section{Statistical analysis}

General linear modelling procedures were used to compare baseline participant characteristics by quintiles of changes in diet cost and to analyse changes in food group consumption according to low and high changes in energy-adjusted diet cost (1st $v$. 5th quintile). ANOVA test and polynomial contrasts were used to determine overall $P$ and $P_{\text {for linear trend, respectively, }}$ for continuous variables with normal distribution, and the Kruskal-Wallis test was used to determine overall $P$ for non-normal distributions. $P_{\text {for linear trend }}$ for categorical variables was obtained by the Mantel-Haenszel linear-by-linear association $\chi^{2}$ test.

Linear regression models were fitted to analyse the association between changes in energy-adjusted diet cost and changes in MDS-rec, energy density, weight and BMI. Two models were fitted. The first included three variables: sex (men/women, dichotomous), age (years, continuous) and the corresponding baseline exposure variable. The second added six variables: smoking (yes/no, dichotomous), energy intake (MJ, continuous), educational level (more than primary school yes/no, dichotomous), LTPA (metabolic equivalents, $\cdot \mathrm{min} / \mathrm{d}$, continuous) and energy under- and over-reporting (both yes/no, dichotomous). The normality assumption of regression models was assessed by the normal probability plot. In addition, linear regression models including secular trends in diet quality as the exposure variables and changes in diet cost were fitted.

Substitution models were fitted to analyse changes in diet quality by the effect of replacing the changes in monetary costs of red meat and sausages, fast food and soft drinks, fish, cereals, dairy products and pastry with the changes in the price of vegetables and fruits. For this purpose, changes in monetary costs of vegetables and fruits were included simultaneously with red meat and sausages, fast food and soft drinks, fish, cereals, dairy products and pastry in multivariate linear regression models. The difference in the coefficients from these models was used to estimate the effect on changes in diet quality indices of replacing a $1 €$ increase in energy-adjusted diet costs of red meat and sausages, fast food and soft drinks, fish, cereals, dairy products and pastry with a $1 €$ increase in vegetables and fruits.

Cubic spline analysis was performed to investigate non-linear associations between changes in the energy-adjusted diet cost and changes in weight and BMI using the 'gam' package in $\mathrm{R}$ version 3.0.2. The assumption of normality in the regression models was assessed using the normal probability plot.

To explore effect modification according to sex, we modelled interaction terms for sex/weight change and sex/BMI change. Differences were considered significant if $P<0.05$. Statistical analyses were performed using SPSS version 18.0 (SPSS Inc.).

\section{Results}

Daily diet cost increased during the follow-up by $35.1 \%$ (online Supplementary Table S1). Substantial differences in energy-adjusted diet cost were observed between low and high diet quality at baseline and at re-examination (online Supplementary Table S1). No significant effect modification by sex was observed $(P>0 \cdot 1)$.

In the bivariate analysis, changes in energy-adjusted diet cost were positively associated with the proportion of women, age, BMI, energy consumption and energy over-reporting (online Supplementary Table S2). The opposite was true for energy under-reporting.

Differences in the changes observed in food group consumption according to a decrease (1st quintile of changes) and an increase (5th quintile of changes) in energy-adjusted dietary costs are shown in online Supplementary Fig. S2. Participants who strongly increased energy-adjusted diet cost increased their consumption of vegetables, fruits, fish and red meat and sausages and decreased the consumption of pastry, cereal products, soft drinks and fast food. The opposite was observed for those participants who decreased energy-adjusted diet cost. The strongest effect was seen for vegetables and fruits.

Diet quality increased with increasing energy-adjusted diet cost (Table 1). Changes in the MDS-rec were directly associated with increasing energy-adjusted diet costs, whereas the opposite was found for energy density (Table 1). The latter showed the strongest association with changes in energyadjusted diet cost.

An increase of $1 €$ in energy-adjusted diet cost was associated with a decrease of $0.3 \mathrm{~kg}$ in body weight and $0.1 \mathrm{~kg} / \mathrm{m}^{2}$ in BMI. These associations were no longer present when the models were adjusted for energy density (Table 2).

Associations between changes in energy-adjusted diet cost and changes in weight and BMI were tested for non-linearity, but no significant evidence was found ( $P$ for curvature of changes in weight and $\mathrm{BMI}=0.47$ and 0.33 , respectively).

Replacing a $1 €$ increase in the energy-adjusted monetary cost of red meat and sausages, fast food and soft drinks, pastry and cereals with $1 €$ increase in vegetables and fruits significantly increased the MDS-rec (Table 3) and decreased energy density.

\section{Discussion}

An increase in the energy-adjusted diet cost predicted a shift to a healthier diet and to better weight management. Diet quality strongly increased when money previously spent on unhealthy food choices such as fast food and pastry was instead spent on vegetables and fruits.

A recently published meta-analysis ${ }^{(5)}$ concluded that healthier diets are more expensive than less healthy diets. The authors found a difference of $\$ 1.54 / 8.36 \mathrm{MJ}$ per $\mathrm{d}$ between extreme quintiles of diet quality, defined by a nutrient-based dietary pattern. The monetary cost of a healthy dietary pattern, defined post boc by cluster analysis, was twice the price of the least healthy pattern in the UK Women's Cohort Study ${ }^{(26)}$. Monsivais et al. ${ }^{(27)}$ reported that strong adherence to the Dietary Approaches to Stop Hypertension diet was $0 \cdot 78 \$ / 8.36 \mathrm{MJ}$ more expensive than low 
Table 1. Association between changes in monetary diet cost and changes in adherence to modified Mediterranean diet score-recommended (MDS-rec) intake and energy density

( $\beta$ Coefficients and $95 \%$ confidence intervals)

\begin{tabular}{|c|c|c|c|c|c|c|}
\hline & \multicolumn{3}{|c|}{ Model $1^{*}$} & \multicolumn{3}{|c|}{ Model $2 \dagger$} \\
\hline & $\beta \ddagger$ & $95 \% \mathrm{Cl}$ & $P$ & $\beta \ddagger$ & $95 \% \mathrm{Cl}$ & $P$ \\
\hline \multicolumn{7}{|l|}{ Scores } \\
\hline \multicolumn{7}{|l|}{ Continuous } \\
\hline$\Delta$ MDS-rec§ & 0.024 & $0.007,0.041$ & 0.006 & 0.042 & $0.025,0.060$ & $<0.001$ \\
\hline$\Delta$ Energy densityll & -1.591 & $-1.703,-1.479$ & $<0.001$ & -1.586 & $-1.699,-1.473$ & $<0.001$ \\
\hline \multicolumn{7}{|l|}{ Quintiles } \\
\hline$\Delta$ MDS-rec§ & 0.049 & $0.013,0.084$ & 0.007 & 0.083 & $0.046,0.199$ & $<0.001$ \\
\hline$\Delta$ Energy densityll & -0.396 & $-0.425,-0.367$ & $<0.001$ & -0.393 & $-0.422,-0.29$ & $<0.001$ \\
\hline \multicolumn{7}{|l|}{ 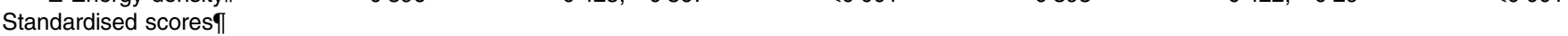 } \\
\hline$\Delta$ MDS-rec§ & 0.067 & $0.019,0.115$ & 0.006 & 0.118 & $0.069,0.167$ & $<0.001$ \\
\hline$\Delta$ Energy densityll & -0.580 & $-0.621,-0.539$ & $<0.001$ & -0.578 & $-0.620,-0.537$ & $<0.001$ \\
\hline
\end{tabular}

* Model 1: adjusted for sex (men/women; dichotomous), age (years; continuous) and baseline energy-adjusted diet cost.

† Model 2: model 1 plus baseline data for smoking (yes/no; dichotomous), energy intake (MJ; continuous), educational level (more than primary school, yes/no; dichotomous), leisure-time physical activity (metabolic equivalents, min/d; continuous) and energy under- and over-reporting (both yes/no; both dichotomous).

$\ddagger$ Linear regression analysis $\beta$ coefficients reflect changes in energy-adjusted diet cost per 1 unit increase in continuous diet quality scores and per 1 quintile increase in categorical diet quality scores.

$\S$ Changes in the MDS-rec.

chere standardised as a Z-value.

If Changes in energy density.

Table 2. Association between changes in energy-adjusted diet cost and changes in body weight and BMI* ( $\beta$ Coefficients and $95 \%$ confidence intervals)

\begin{tabular}{|c|c|c|c|c|c|c|c|c|c|}
\hline & \multicolumn{3}{|c|}{ Model 1† } & \multicolumn{3}{|c|}{ Model $2 \ddagger$} & \multicolumn{3}{|c|}{ Model 3§ } \\
\hline & $\beta$ & $95 \% \mathrm{Cl}$ & $P$ & $\beta$ & $95 \% \mathrm{Cl}$ & $P$ & $\beta$ & $95 \% \mathrm{Cl}$ & $P$ \\
\hline $\begin{array}{l}\Delta \text { Weight }(\mathrm{kg}) \| \\
\Delta \mathrm{BMI}\left(\mathrm{kg} / \mathrm{m}^{2}\right)\end{array}$ & $\begin{array}{l}-0.30 \\
-0.10\end{array}$ & $\begin{array}{l}-0.53,-0.07 \\
-0.19,-0.01\end{array}$ & $\begin{array}{l}0.01 \\
0.03\end{array}$ & $\begin{array}{l}-0.29 \\
-0.10\end{array}$ & $\begin{array}{l}-0.52,-0.07 \\
-0.18,-0.01\end{array}$ & $\begin{array}{l}0.02 \\
0.04\end{array}$ & $\begin{array}{r}-0.10 \\
0.00\end{array}$ & $\begin{array}{l}-0.38,0.19 \\
-0.11,0.10\end{array}$ & $\begin{array}{l}0.51 \\
0.99\end{array}$ \\
\hline
\end{tabular}

* Multiple linear regression analysis. $\beta$ Coefficients reflect changes in body weight and BMI per $1 € / 8.36 \mathrm{MJ}$ increase in diet cost.

† Model 1: adjusted for sex (men/women; dichotomous), age (years; continuous) and baseline scores.

¥ Model 2: includes additionally baseline data for smoking (yes/no; dichotomous), energy intake (MJ; continuous), educational level (more than primary school yes/no; dichotomous), leisure-time physical activity (metabolic equivalents, $\cdot \mathrm{min} / \mathrm{d}$; continuous) and energy under- and over-reporting (both yes/no; dichotomous).

$\S$ Model 3: includes additionally $\Delta$ energy density (continuous).

II Changes in body weight.

I Changes in BMI.

Table 3. Association between 10-year changes in diet quality and replacement of 1€/8.36 MJ increased consumption of fast food and soft drinks, pastry, red meat and sausages, fish and seafood, cereals and dairy products, with $1 € / 8.36 \mathrm{MJ}$ increase in fruits and vegetables*

( $\beta$ Coefficients and $95 \%$ confidence intervals)

\begin{tabular}{lcccc}
\hline & \multicolumn{2}{c}{ MDS-rec } & \multicolumn{2}{c}{ Energy density } \\
\cline { 2 - 3 } & \multicolumn{1}{c}{$\beta$} & $95 \% \mathrm{Cl}$ & $\beta$ & $95 \% \mathrm{Cl}$ \\
\cline { 2 - 5 } Fast food and soft drinks & 2.98 & $1.58,4.37$ & -0.36 & $-0.533,-0.187$ \\
Pastry & 3.94 & $1.08,6.81$ & -1.32 & $-1.67,-0.97$ \\
Red meat and sausages & 1.33 & $1.04,1.61$ & -0.12 & $-0.15,-0.08$ \\
Fish and seafood & -0.28 & $-0.73,0.17$ & -0.01 & $-0.04,0.02$ \\
Cereals & 0.47 & $0.15,0.79$ & -0.21 & $-0.25,-0.17$ \\
Dairy products & -0.79 & $-1.15,-0.35$ & 0.02 & $-0.03,0.07$ \\
\hline
\end{tabular}

MDS-rec, modified Mediterranean diet score-recommended intake.

* Linear regression analysis adjusted for sex (men/women; dichotomous), age (years; continuous) and baseline data of smoking (yes/no; dichotomous), energy intake (MJ; continuous), educational level (more than primary school yes/no; dichotomous), leisure-time physical activity (metabolic equivalents, min/d; continuous) and energy under- and over-reporting (both yes/no; dichotomous). $\beta$ Coefficients reflect changes in diet quality scores of replacement of $1 € / 8.36 \mathrm{MJ}$ increased consumption of fast food and soft drinks, pastry, red meat and sausages, fish and seafood, cereals and dairy products with $1 € / 8.36 \mathrm{MJ}$ increase in fruits and vegetables.

adherence to this dietary pattern. In the present study, the energy-adjusted diet cost for high diet quality was $2 \cdot 95 €(\$ 3.33) / \mathrm{d}$ higher than low diet quality; this amounts to $1076 €(\$ 1215) /$ year for one person who chooses high diet quality. One might hypothesise that this would negatively influence healthy food choices, particularly in low-income families. 
We used two conceptually different indices to measure overall diet quality: food-based and energy density, which we have shown to be good indicators of diet quality in the present population $^{(9,10)}$. Our prospective results indicate that reducing diet cost has detrimental effects on diet quality. This was true for both indicators of diet quality, underlining the robustness of our data.

In the present study, an increase in energy-adjusted diet cost of $1 €$ represented a $54.5 \%$ difference between the 2 nd and 4 th quintiles in energy-adjusted diet cost changes. The change from a strong decrease to a strong increase in diet quality measured by adherence to the Mediterranean diet and energy density was associated with an increase of $0.42 €$ and $1.98 €$ in the energy-adjusted diet cost, respectively. For both diet quality scores, the percentage difference and percentage increase in energy-adjusted diet cost between the strong decrease and strong increase was 133 and $400 \%$, respectively.

The price of healthy foods increased to a greater extent than that of less healthy foods in Spain between 2000 and $2010^{(8)}$, and price is an important determinant for food choices ${ }^{(4)}$. Individuals and families facing economic constraints may be especially likely to reduce their consumption of more expensive foods, regardless of their contribution to diet quality. In addition, it is not surprising that a strong decrease in diet cost in the present study was concomitant with a dramatic decrease in the consumption of fruits and vegetables.

On the other hand, fast food and soft drinks consumption increased in participants who greatly reduced their diet cost. This is of particular concern because soft drink and fast food consumptions are associated with less healthy dietary patterns and weight management in the present population ${ }^{(28)}$. Moreover, low diet quality is responsible for $17 \%$ of disabilityadjusted life years in the USA ${ }^{(29)}$. Low consumption of fruits and vegetables is one characteristic of this low diet quality. Our substitution models convincingly show the positive effect on diet quality of replacing $1 €(\$ 0 \cdot 86)$ increments of dietary costs in pastry and soft drinks and fast food with $1 €$ increases in fruits and vegetables. These data underline the paramount role of fruit and vegetable consumption in a healthy diet. Moreover, our data raise the question of food price intervention using tax policy and subsidies. Evidence indicates that a rise in prices of unhealthy foods and a price reduction for healthier alternatives improve overall diet quality ${ }^{(4,30,31)}$.

Following the Mediterranean dietary pattern and low energy-dense diets have been frequently associated with better weight management and reduced risk of obesity ${ }^{(11,18,32)}$. Therefore, and based on the present results, we hypothesised that changes in diet cost would affect body weight. Our analysis showed a direct relationship between a decrease in diet cost and weight gain. This association was mainly explained by diet quality; adjusting for changes in diet quality strongly attenuated the impact of increased diet cost on weight gain.

This study has both limitations and strengths. Owing to the nature of observational studies, causal relationships cannot be drawn. Furthermore, all the dietary instruments that measure past food intake are vulnerable to random and systematic measurement errors. Although the 10-year loss to follow-up of $19.7 \%$ in the present study can be considered acceptable, there was some evidence of selection bias among the participants who completed the follow-up in that they were generally younger and more likely to be female. Variation of monetary cost of food due to regions, seasons, and types of establishment where the food was purchased is a potential bias for the analysis of the impact of diet cost on diet quality. In the present study, we used yearly averages of food prices across multiple regions of Spain, which somewhat reduced this limitation. Furthermore, we do not have data on food consumption away from home. Our analysis was based on the assumption that most foods consumed were prepared at home. Indeed the findings of this study may not hold for those who frequently eat away from home. The strengths of the present study include its population-based design, long-term follow-up and the availability of body weight and validated lifestyle measurements at baseline and follow-up.

Results of the present study are in line with previous findings showing that healthy diets are considerably more expensive than unhealthy diets. Our prospective evidence indicates that a worsening of overall diet quality and weight development was related to a decrease in diet cost. This finding is of importance for health policy because it underlines the need to promote healthy diets that are accessible for all income levels, with implications for food pricing, agricultural and consumer subsidy programmes and tax policies.

\section{Acknowledgements}

The authors appreciate the English revision by Elaine Lilly, $\mathrm{PhD}$ (Writer's First Aid).

This work was supported by grants from Instituto de Salud Carlos III FEDER (CB06/02/0029), and AGAUR (2014 SGR 240). CIBER Epidemiology and Public Health and CIBER Physiopathology of Obesity and Nutrition are an initiative of the Instituto de Salud Carlos III, Madrid, Spain.

H. S., L. S. M. and R. E. designed the research; H. S., L. S. M., I. S., M. I. P., M. F. and R. E. conducted the research; H. S. and I. S. analysed the data; and H. S. wrote the manuscript and had primary responsibility for the final content. All the authors read and approved the final version of the manuscript.

The authors declare that they have no conflicts of interest.

\section{Supplementary material}

For supplementary material/s referred to in this article, please visit http://dx.doi.org/doi:10.1017/S0007114515005048

\section{References}

1. Mozaffarian D, Appel LJ \& Van Horn L (2011) Components of a cardioprotective diet: new insights. Circulation 123, 2870-2891.

2. O'Neil A, Quirk SE, Housden S, et al. (2014) Relationship between diet and mental health in children and adolescents: a systematic review. Am J Public Health 104, e31-e42.

3. Beaglehole R, Bonita R, Horton R, et al. (2011) Priority actions for the non-communicable disease crisis. Lancet $\mathbf{3 7 7}$, $1438-1447$.

4. French SA (2003) Pricing effects on food choices. J Nutr 133 , 841S-843S. 
5. Rao M, Afshin A, Singh G, et al. (2013) Do healthier foods and diet patterns cost more than less healthy options? A systematic review and meta-analysis. BMJ Open 3, e004277.

6. Beydoun MA, Shroff MR, Chen X, et al. (2011) Serum antioxidant status is associated with metabolic syndrome among U.S. adults in recent national surveys. J Nutr 141, 903-913.

7. FAOSTAT (2015) Food and Agriculture Organization of the United Nations, Statistics Division. http://faostat3.fao.org/ download/P/CP/E (accessed October 2015).

8. Información de precios (Base de datos) (2015) Spanish Ministry of Economy and Competitiveness. http://www.comercio. gob.es/es-ES/comercio-interior/Precios-y-Margenes-Comerciales/ Informacion-de-precios-(bases-de-datos)/Paginas/Precios-OrigenDestino-.aspx (accessed March 2012).

9. Schroder H, Covas M, Elosua R, et al. (2008) Diet quality and lifestyle associated with free selected low-energy density diets in a representative Spanish population. Eur J Clin Nutr 62 , 1194-1200.

10. Schroder H, Vila J, Marrugat J, et al. (2008) Low energy density diets are associated with favorable nutrient intake profile and adequacy in free-living elderly men and women. J Nutr $\mathbf{1 3 8}$, 1476-1481.

11. Savage JS, Marini M \& Birch LL (2008) Dietary energy density predicts women's weight change over 6 y. Am J Clin Nutr $\mathbf{8 8}$, 677-684.

12. Vernarelli JA, Mitchell DC, Rolls BJ, et al. (2015) Dietary energy density is associated with obesity and other biomarkers of chronic disease in US adults. Eur J Nutr 54, 59-65.

13. Benitez-Arciniega AA, Mendez MA, Baena-Diez JM, et al. (2011) Concurrent and construct validity of Mediterranean diet scores as assessed by an FFQ. Public Health Nutr 14, 2015-2021.

14. Schroder H, Covas MI, Marrugat J, et al. (2001) Use of a three-day estimated food record, a 72-hour recall and a foodfrequency questionnaire for dietary assessment in a Mediterranean Spanish population. Clin Nutr 20, 429-437.

15. Sofi F, Macchi C, Abbate R, et al. (2014) Mediterranean diet and health status: an updated meta-analysis and a proposal for a literature-based adherence score. Public Health Nutr 17, 2769-2782.

16. Estruch R, Ros E, Salas-Salvadó J, et al. (2013) Primary prevention of cardiovascular disease with a Mediterranean diet. N Engl J Med 368, 1279-1290.

17. Schröder H, Salas-Salvadó J, Martínez-González MA, et al. (2014) Baseline adherence to the Mediterranean diet and major cardiovascular events: Prevención con Dieta Mediterránea trial. JAMA Intern Med 174, 1690-1692.

18. Funtikova AN, Benitez-Arciniega AA, Gomez SF, et al. (2014) Mediterranean diet impact on changes in abdominal fat and 10 -year incidence of abdominal obesity in a Spanish population. Br J Nutr 111, 1481-1487.
19. Ledikwe JH, Blanck HM, Khan LK, et al. (2005) Dietary energy density determined by eight calculation methods in a nationally representative United States population. $J$ Nutr 135, 273-278.

20. Johnson L, Wilks DC, Lindroos AK, et al. (2009) Reflections from a systematic review of dietary energy density and weight gain: is the inclusion of drinks valid? Obes Rev 10, 681-692.

21. Mendez MA, Popkin BM, Buckland G, et al. (2011) Alternative methods of accounting for underreporting and overreporting when measuring dietary intake-obesity relations. $\mathrm{Am} \mathrm{J}$ Epidemiol 173, 448-458.

22. Mifflin MD, St Jeor ST, Hill LA, et al. (1990) A new predictive equation for resting energy expenditure in healthy individuals. Am J Clin Nutr 51, 241-247.

23. Black AE (2000) Critical evaluation of energy intake using the Goldberg cut-off for energy intake: basal metabolic rate. A practical guide to its calculation, use and limitations. Int J Obes Relat Metab Disord 24, 1119-1130.

24. Elosua R, Garcia M, Aguilar A, et al. (2000) Validation of the Minnesota leisure time physical activity questionnaire in Spanish women. Investigators of the MARATDON Group. Med Sci Sports Exerc 32, 1431-1437.

25. Elosua R, Marrugat J, Molina L, et al. (1994) Validation of the Minnesota leisure time physical activity questionnaire in Spanish men. The MARATHOM Investigators. Am J Epidemiol 139, 1197-1209.

26. Morris MA, Hulme C, Clarke GP, et al. (2014) What is the cost of a healthy diet? Using diet data from the UK Women's Cohort Study. J Epidemiol Community Health 68, 1043-1049.

27. Monsivais P, Rehm CD \& Drewnowski A (2013) The DASH diet and diet costs among ethnic and racial groups in the United States. JAMA Intern Med 173, 1922-1924.

28. Schroder H, Fito M \& Covas MI (2007) Association of fast food consumption with energy intake, diet quality, body mass index and the risk of obesity in a representative Mediterranean population. BrJ Nutr 98, 1274-1280.

29. Collaborators USBoD (2013) The state of US health, 1990-2010: burden of diseases, injuries, and risk factors. JAMA 310, 591-608.

30. Epstein LH, Dearing KK, Paluch RA, et al. (2007) Price and maternal obesity influence purchasing of low- and highenergy-dense foods. Am J Clin Nutr 86, 914-922.

31. Herman DR, Harrison GG, Afifi AA, et al. (2008) Effect of a targeted subsidy on intake of fruits and vegetables among low-income women in the Special Supplemental Nutrition Program for Women, Infants, and Children. Am J Public Health 98, 98-105.

32. Beunza JJ, Toledo E, Hu FB, et al. (2010) Adherence to the Mediterranean diet, long-term weight change, and incident overweight or obesity: the Seguimiento Universidad de Navarra (SUN) cohort. Am J Clin Nutr 92, 1484-1493. 